\title{
Consequências da Ingestão Moderada de Etanol na Discriminação de Notas Musicais
}

\section{Consequences of Moderate Intake of Ethanol in the Perception of Musical Notes}

\author{
Jandilson Avelino da Silva*, ${ }^{a}$, Melyssa Kellyane Cavalcanti Galdino ${ }^{a}$, \\ Maria Lúcia de Bustamante $\operatorname{Simas}^{b} \&$ Natanael Antonio dos Santos ${ }^{a}$ \\ ${ }^{a}$ Universidade Federal da Paraiba, João Pessoa, PB, Brasil \\ $\&{ }^{b}$ Universidade Federal de Pernambuco, Recife, PE, Brasil
}

\begin{abstract}
Resumo
O objetivo deste estudo foi avaliar se existe relação entre a ingestão moderada de etanol e a discriminação das notas musicais RÉ, FÁ, e LÁ, em adultos jovens. Essas notas apresentam frequências fundamentais respectivamente de $293,665 \mathrm{~Hz}, 349,228 \mathrm{~Hz}$ e $440 \mathrm{~Hz}$, de temperamento igual, escolhidas devido estarem em localização central na escala musical ocidental padrão. Participaram do estudo 40 voluntários de 18 a 29 anos, não músicos, saudáveis, não usuários de fármacos ou outras substâncias tóxicas, exceto o etanol de forma moderada e aguda. Por meio do software "Psysounds", criado especificamente para esse estudo, esses voluntários tiveram de escolher, entre duas notas musicais, uma das notas teste apresentada inicialmente. Os dados foram tratados com um teste X2 de aderência para comparar as frequências de acertos das notas musicais de teste nas condições de ingestão e de não ingestão de álcool. As análises mostraram diferenças significantes nas frequências de acertos para todas as notas utilizadas, sugerindo que a ingestão de etanol prejudica a discriminação das notas musicais RÉ, FÁ e LÁ.
\end{abstract}

Palavras-chave: Discriminação de tons, notas musicais, bebidas alcoólicas.

\begin{abstract}
The aim of this study was to evaluate if there is relationship between moderate intake of ethanol and the perception of the musical notes D, F, and A in young adults. These notes have fundamental frequencies of $293.665 \mathrm{~Hz}, 349.228 \mathrm{~Hz}$, and $440 \mathrm{~Hz}$, respectively, equal temperament and were chosen because they are in the central location in the standard western musical scale. The study included 40 volunteers from 18 to 29 years old, non-musicians, healthy, non-users of drugs or other toxic substances, except moderate and acute intake of ethanol. By the "Psysounds" software, created specifically for this study, the volunteers had to choose between two musical notes one of the test notes initially presented. The data were treated with X2 test of adherence to compare the frequency of correct answers regarding the musical notes in the conditions of intake and non-intake of alcohol. The analysis showed significant differences in the frequencies of correct answers for all the notes used, suggesting that ethanol ingestion impairs the discrimination of musical notes $\mathrm{D}, \mathrm{F}$ and $\mathrm{A}$.

Keywords: Pitch perception, musical notes, alcoholic beverages.
\end{abstract}

O uso de bebidas alcoólicas é popular e constitui um problema relevante no cenário mundial. Inclusive, há quem considere o álcool um problema de saúde pública (Vieira, Serafim, \& Saffi, 2007) devido aos prejuízos pessoais e socioeconômicos associados ao seu consumo e até mesmo ao aumento da violência urbana provavelmente ocasionados por alterações perceptuais, principalmente as visuais (Bastos, Caiaffa, Rossi, Vila, \& Malta, 2007; Cavalcanti \&

\footnotetext{
* Endereço para correspondência: Departamento de Psicologia, Universidade Federal da Paraíba, Laboratório de Percepção, Neurociências, e Comportamento, Campus I, Cidade Universitária, Avenida Castelo Branco, Bairro Castelo Branco III, João Pessoa, PB, Brasil 58052-900. E-mail: jandilsonsilva@gmail.com Apoio CNPq: Processo 303822/2010-2
}

Santos, 2008; Galdino, Mendes, Vieira, Simas, \& Santos, 2011; Galdino, Silva, Santos, \& Simas, 2010).

No entanto, a ingestão de álcool tem sido associada também a alterações na amplitude do potencial de respostas de neurônios do córtex auditivo, reduzindo, dessa forma, a sensibilidade para detecção de frequências sonoras (Pearson, Dawe, \& Timney, 1999). O estudo de Pearson (1997), por exemplo, realizado com seis participantes (divididos igualmente entre os sexos masculino e feminino), utilizou tons puros de frequências de 100 a $3200 \mathrm{~Hz}$ que se alteravam de forma ascendente e descendente. Essas mudanças deveriam ser identificadas pelos participantes em um teste psicofísico de diferenciação de sons. Demonstrou-se nesse estudo que a ingestão de álcool a $0,08 \%$ BAC (Blood Alcohol Concentration/Concentração de Álcool no Sangue) 
pode ocasionar alterações na discriminação de sons com frequências acima de $1000 \mathrm{~Hz}$. O BAC é medido por meio do hálito dos indivíduos e pode ser avaliado por etilômetro, um aparelho que mede a presença de álcool no ar alveolar $\mathrm{em} \mathrm{mg} / \mathrm{lBrac}$ que pode ser transformado em porcentagem BAC por meio da divisão de seu valor por 5 (cinco).

Em outro estudo, de Jääskeläinen et al. (2000), realizado com dez participantes, de 20 a 28 anos, avaliados por exames de eletroencefalografia (EEG) em uma tarefa de atenção seletiva, tinha-se que atentar para um som apresentado à uma determinada orelha enquanto ignorava o som apresentado à uma outra. Os participantes tinham que detectar um som desviante de um tom padrão atendido. Um padrão de $300 \mathrm{~Hz}$ e um tom desviante de $330 \mathrm{~Hz}$ foram apresentados à orelha esquerda, e um padrão de $1000 \mathrm{~Hz}$ e um desviante $1100 \mathrm{~Hz}$ para a orelha direita dos indivíduos. Os resultados de Jääskeläinen et al. apresentaram supressão auditiva transitória decorrente da ingestão de álcool à $0,05 \%$ BAC.

He et al. (2013) avaliaram os efeitos do álcool no processamento pré-atentivo da audição em quatro de suas características psicoacústicas: frequência, intensidade, localização e duração. No entanto, esses autores estudaram esses aspectos por meio do Mismatch Negativity (MMN), um tipo de potencial evocado auditivo de longa latência que proporciona um índice objetivo da discriminação e da memória sensorial auditiva. He et al. concluíram que a ingestão de álcool altera todas as características avaliadas, sendo que a frequência é a característica mais afetada.

Kahkonen, Rossi, e Yamashita (2005), do mesmo modo, estudaram se o álcool prejudica o processamento auditivo de mudanças de frequência por meio de EEG, nesse caso conjugado a magnetoencefalografia (MEG). Os estímulos que variaram sempre entre $560 \mathrm{~Hz}$ e 665 $\mathrm{Hz}$, mediante um som padrão de $700 \mathrm{~Hz}$, foram apresentados separadamente para as orelhas esquerda e direita de 11 indivíduos que haviam ingerido $0,8 \mathrm{~g} / \mathrm{kg}$ de álcool combinado com suco de laranja. Os resultados mostraram prejuízos para o processamento dos sons nos diferentes hemisférios.

Esses estudos, embora sejam importantes para a área, e para esse estudo em específico, por servirem como base de descrição das possíveis alterações auditivas que as bebidas alcoólicas podem ocasionar, com exceção do estudo de Pearson (1997), restringem-se a estudos eletrofisiológicos. Estudos psicofísicos, como o do presente manuscrito podem contribuir para a futura elaboração de avaliações mais rápidas dos efeitos de substâncias no processamento auditivo. As tarefas de discriminação sonora que o presente estudo utilizou são relevantes para o entendimento de procedimentos gerais de interação social como o reconhecimento dos diversos sons, bem como de suas fontes. Dessa forma, a interferência do álcool na audição podendo dificultar a relação com o meio, seja no reconhecimento de vozes ou transformando o entendimento prosódico das falas humanas, é uma importante justificativa para realização de estudos dessa natureza (Pearson, 1997).
Além dos aspectos socioeconômicos, comportamentais e fisiológicos associados ao álcool, esse estudo também foi motivado pela carência de estudos psicofísicos que avaliem os efeitos específicos da ingestão moderada de álcool na discriminação de sons mais significativos socialmente, isto é, notas musicais com tons complexos e timbres reconhecíveis ao invés de tons puros tipicamente mais utilizados pela literatura. Estudos a respeito do processamento musical de forma mais global podem ser encontrados, como os de Engels, Poelen, Spijkerman, e Ter Bogt (2012), e de Kornreich et al. (2013). No entanto, esses estudos não podem ser diretamente utilizados como base de comparação para o presente estudo por focarem na influência de aspectos emocionais ou do gênero musical no nível de ingestão de álcool. Além disso, o primeiro trata-se da avaliação de alcoolistas em recuperação e não da ingestão experimental aguda de álcool. Já o segundo tem limitações relativas a questões metodológicas básicas como a falta de controle do limite alcoólico no sangue e do efeito de expetativa por meio do uso de placebo.

Nesse contexto, esse estudo objetivou utilizar as notas musicais RÉ, FÁ e LÁ, com timbre sintético de piano, pertencentes à quarta oitava de uma escala musical padrão. Essas notas apresentam frequências fundamentais respectivamente de $293,665 \mathrm{~Hz}, 349,228 \mathrm{~Hz}$ e $440 \mathrm{~Hz}$, de temperamento igual, escolhidas devido estarem em localização central em uma escala musical ocidental padrão. Pretende-se avaliar se existe alteração na discriminação de tais notas musicais em adultos jovens com e sem a ingestão moderada de álcool $(0,08 \%$ BAC). Essa concentração em porcentagem de álcool por litro de sangue, já ocasiona interferências no comportamento motor dos indivíduos (Cavalcanti \& Santos, 2008; Galdino et al., 2010), mas não se pode concluir por meio dos poucos estudos existentes se as alterações também se dão no âmbito perceptivo e cognitivo. Inclusive, existe uma grande variabilidade nesse limite alcoólico utilizado como padrão entre os estudos. Contudo, esse estudo trata-se de uma pesquisa exploratória na qual não se tem a intenção de extrapolar que essas notas musicais utilizadas possam ser representativas de toda discriminação auditiva humana de alturas possível em pessoas alcoolizadas.

\section{Método}

\section{Aspectos Éticos}

Esse estudo foi aprovado por um Comitê de Ética local, sob o protocolo $\mathrm{n}^{\circ}$. 075/10. A participação voluntária na pesquisa aconteceu mediante a assinatura de um termo de consentimento livre e esclarecido, no qual os voluntários foram informados sobre os objetivos do trabalho, seguindo todas as recomendações regidas pela Resolução Brasileira $n^{\circ}$. 196/96 do Conselho Nacional de Saúde.

\section{Caracterização da Amostra}

Critérios de Inclusão e Exclusão. Os critérios de inclusão na amostra foram: ser adulto, tendo mais de 18 de 
Silva, J. A., Galdino, M. K. C., Simas, M. L. B. \& Santos, N. A. (2015). Consequências da Ingestão Moderada de Etanol na Discriminação de Notas Musicais.

idade; não ter experiência de estudo formal com música, como por exemplo, não tocar qualquer instrumento; estar em boa saúde física e mental; não fazer uso de fármacos ou de outras substâncias tóxicas exceto de etanol; fazer uso moderado contínuo de bebidas alcoólicas sem nenhuma intercorrência pessoal e/ou familiar (como dependência à substância ou problemas legais relacionados ao uso da mesma). Esses critérios foram avaliados pelo questionário sócio demográfico criado pelos próprios pesquisadores para o estudo e pelo Alcohol Use Disorder Identification Test (AUDIT; Piccinelli et al., 1997), que serão descritos na seção seguinte de instrumentos e equipamentos. Todos os voluntários que não atenderam aos critérios de inclusão foram excluídos.

Descrição da Amostra. Participaram do estudo 40 estudantes universitários brasileiros de graduação e de pós-graduação (sendo 20 homens e 20 mulheres). Os voluntários encontravam-se clinicamente saudáveis e sem queixas auditivas, já tinham feito uso de bebidas alcoólicas, não eram músicos, e estavam na faixa etária de 18 a 29 anos de idade (Mulheres: 21,95 $\pm 3,14$ e Homens: 21,65 $\pm 2,73$ ).

Considerando o total de voluntários, na sua maioria eram destros $(97,5 \%)$, brancos $(55 \%)$ e estavam fazendo graduação $(90 \%)$. O curso de maior representatividade foi o de Psicologia (82,5\%), tendo maior participação os estudantes do primeiro $(32,5 \%)$, quarto $(17,5 \%)$, sexto $(10 \%)$ e oitavo $(10 \%)$ semestres. A maior parte da amostra era composta por solteiros $(97,5 \%)$, católicos $(55 \%)$, com renda de três a cinco salários mínimos $(52,5 \%)$.

A maioria dos voluntários tomavam bebidas alcoólicas há $3(22,5 \%), 4(17,5 \%), 5(12,5 \%), 6(10 \%)$ e $7(10 \%)$ anos, sendo que a maior parte deles bebia pelo menos uma $(80 \%)$ e duas vezes $(15 \%)$ por semana. Estes costumavam beber em sua maioria cerveja $(57,5 \%)$, vodka $(20 \%)$ ou vinho $(12,5 \%)$, sendo que na maior parte das vezes bebiam $4(22,5 \%), 5(22,5 \%), 2(17,5 \%), 6(10 \%)$ e $8(10 \%)$ copos de cerveja ou vinho ou doses de vodka.

\section{Instrumentos e Equipamentos Utilizados na Pesquisa}

Um questionário composto por questões sócio-demográficas (como idade, sexo, escolaridade, entre outras) e clínicas (comprometimentos físicos e psicológicos, uso de substâncias tóxicas, entre outras), com o objetivo de caracterização da amostra.

O Alcohol Use Disorder Identification Test (AUDIT; Piccinelli et al., 1997), uma escala com 10 itens que abrangem três domínios teóricos: 1- Frequência do consumo de álcool (Ex.: "Com que frequência você consome alguma bebida alcoólica?"); 2- Dependência do consumo de álcool (Ex.: "Com que frequência você achou que não seria capaz de controlar a quantidade de bebida depois de começar?"); 3- Consequências negativas do consumo de álcool (Ex.: "Com que frequência você sentiu culpa ou remorso depois de beber?").

Dosagem, um software elaborado pelos pesquisadores para calcular a quantidade de bebida alcoólica a ser ingerida levando em consideração o sexo dos voluntários para se atingir o valor da porcentagem BAC pretendida no estudo. No Dosagem, cálculos diferentes são realizados para homens e mulheres baseando-se na quantidade de água corporal dos mesmos a partir de adaptações das fórmulas sugeridas por Brick (2006). As variáveis peso e altura são utilizadas para definir a quantidade de álcool a ser ingerida pelas mulheres, e no caso dos homens o valor da idade também deve ser inserido. A estimativa da quantidade de álcool é computada na seguinte sequência:

1. Cálculo da quantidade total de água corporal, sendo, (a) fórmula para os homens:

$2.44-(0,09516 \times$ idade $)+(0,1074 \times$ altura $)+$ $(0,3362 \times$ peso). (Eq. 1$)$

(b) fórmula para mulheres:

$-2,097+(0,1069 \mathrm{x}$ altura $)+(0,2466 \mathrm{x}$ peso $)$. (Eq. 2)

2. Cálculo da quantidade em gramas de álcool a ser ingerida para o valor em BAC desejado, sendo a fórmula,

$80+(15 \times 0,75) \times$ quantidade total de água corporal/80,65. (Eq. 3)

3. Transformação da quantidade em gramas para a quantidade em $\mathrm{ml}$ de álcool por meio de uma regra de três simples relacionando $1 \mathrm{ml}$ à $0,8 \mathrm{~g}$, já que se pretende chegar ao teor de $0,08 \mathrm{BAC}$;

4. Transformação da quantia em ml para a quantia de vodka a ser ingerida, por meio de uma regra de três simples relacionando $1.000 \mathrm{ml} \mathrm{a} 400 \mathrm{ml}$, considerando a porcentagem alcoólica da vodka em $40 \%$.

O PsySounds, um software programado na linguagem JAVA, gerador de notas musicais por meio do sequenciamento conjugado de áudio e MIDI (Musical Instrument Digital Interface/Interface Digital para Instrumentos Musicais) especificamente desenvolvido pelos pesquisadores, para a realização deste experimento. Esse programa foi processado por um micro-computador desktop com sistema operacional "Windows $X P$ ", ao qual foram conectados uma placa de som externa da marca "Creative", modelo "X-Fi Surround USB 5.1 Sound Blaster" e um mouse, para indicação das respostas dos voluntários aos estímulos apresentados; o PsySounds foi programado em linguagem Java e seus estímulos gerados simulam as sete oitavas de um piano, dentro das quais pode ser escolhida qualquer uma das sete notas musicais de uma escala padrão (DÓ, RÉ, MI, FÁ, SOL, LÁ E SI). A janela de abertura do programa apresenta a possibilidade da escolha visual na tela de um monitor (uma espécie de pré-programação) de notas musicais à direita (nota teste, que se trata da nota avaliada, ex.: RÉ) e à esquerda (notas distratoras, que se tratam das notas a serem comparadas com a nota avaliada, ex.: DÓ E MI) pelo experimentador. Depois de selecionadas, e iniciada a sessão experimental, as notas musicais foram apresentadas ao participante por um segundo em pares de notas teste e distratora, em ordem aleatória, separadas por 
um intervalo de tempo de também um segundo. Em cada alternativa apenas uma nota distratora foi apresentada em comparação a nota teste.

Monitor de vídeo CRT de 19 polegadas da marca "LG" para apresentação dos estímulos e manipulação dos softwares utilizados;

Dois fones de ouvidos, sendo um para o experimentador, da marca "Nokia", modelo "WH102", e outro para o participante, da marca "Sennheiser", modelo "HD 205". $\mathrm{O}$ fone do experimentador era mais simples e utilizado apenas para acompanhamento e controle da apresentação dos estímulos. $\mathrm{O}$ fone do participante tinha por objetivo apresentar com a maior fidedignidade possível o som apresentado. Foi também utilizado um adaptador para a conexão dos fones à placa de som;

Uma venda de tecido que foi colocada sobre os olhos dos voluntários, para evitar que estimulações visuais interferissem nas suas respostas auditivas;

Um etilômetro digital da marca "Instruterm", modelo "BFD-50" que permitiu determinar a quantidade de álcool no sangue. Para a realização das medidas o indivíduo deveria tomar bastante fôlego e assoprar em um bocal descartável presente no lado esquerdo do aparelho.

\section{Estímulos Utilizados}

Para este estudo, foram escolhidas para cada participante em uma sessão experimental as notas RÉ, FÁ e LÁ da quarta oitava com frequências sonoras respectivamente de $293,665 \mathrm{~Hz}, 349,228 \mathrm{~Hz}$ e $440 \mathrm{~Hz}$, de temperamento igual (razão de semitom $2^{1 / 12}: 1$ ) na opção "nota teste", em comparação com as duas notas mais próximas da escala a que ela pertencia, podendo, dessa forma, variar entre as notas DÓ, MI, SOL ou SI, de frequências sonoras respectivas de 261, $63 \mathrm{~Hz}, 329,63 \mathrm{~Hz}, 392 \mathrm{~Hz}$ 493, $88 \mathrm{~Hz}$, como apresentado na Tabela 1, a seguir. O som dessas notas era formado por tom complexo com timbre de piano. A escolha da quarta oitava foi devido a ela localizar-se na porção central das oitavas possíveis dentro das escalas musicais, fato que fez a ISO (International Organization for Standardization/ Organização Internacional para Padronização) considerar o padrão de $440 \mathrm{~Hz}$ (correspondente a nota LÁ central), como referência para afinação de qualquer instrumento musical (Ver Silva, Galdino, Gadelha, Andrade, \& Santos, 2013). A escolha das notas em específico se deu pelo fato de que elas, considerando apenas as teclas brancas de um piano, estão intercaladas entre as notas dos extremos da oitava utilizada, e por dessa forma abarcar a frequência musical padrão $(440 \mathrm{~Hz})$. Além disso, as teclas brancas em sequência são as mais conhecidas pela população geral, embora não tenham intervalos musicais iguais entre elas (a saber, um ou dois semitons entre notas).

\section{Procedimentos}

Antes de cada sessão experimental o participante deveria responder a um questionário, no qual foram feitas indagações sócio-demográficas, como idade, sexo, latera-
Tabela 1

Conjunto de Notas Testes e Distratoras em Quarta Oitava Utilizadas no Experimento de Discriminação de Notas Musicais

\begin{tabular}{cc}
\hline Nota Teste & Nota Distratora \\
\hline RÉ & DÓ OU MI \\
FÁ & MI OU SOL \\
LÁ & SOL OU SI \\
\hline
\end{tabular}

lidade, raça, escolaridade, profissão, estado civil, renda, religião e habilidades musicais. O questionário continha ainda indagações de natureza clínica, relacionadas à saúde física e psicológica geral do participante, principalmente da saúde auditiva, e também ao uso de substâncias como tabaco, café, bebidas alcoólicas e fármacos.

As luzes do ambiente ficaram desligadas no momento do experimento. As medidas obtidas pelas respostas dos participantes foram realizadas por meio do método psicofísico de escolha forçada entre duas alternativas temporais (adaptado dos estudos de percepção visual de Cornsweet, 1970; Levitt, 1971).

Em cada sessão experimental foram apresentados aleatoriamente para cada participante três grupos de 20 pares de estímulos (estímulo de teste e estímulo distrator), sendo cada grupo equivalente a uma das notas utilizadas no estudo como padrão (RÉ, FÁ ou LÁ). A tarefa dos voluntários foi a de escolher sempre o estímulo de teste, a nota musical padronizada para cada grupo de estímulos, entre as duplas de sons apresentados. Essa nota teste foi sempre apresentada isoladamente ao participante por cinco vezes consecutivas antes que a apresentação das duplas de estímulos fosse iniciada.

Os estímulos distratores foram sempre duas notas musicais diferentes da escolhida como teste. Esses estímulos variavam randomicamente, em igual proporção, entre as duas notas vizinhas a cada uma das notas teste, sendo uma localizada à direita e outra à esquerda, considerando apenas as notas musicais correspondentes às teclas brancas de um piano, simuladas no Psysounds. Ou seja, para a nota musical RÉ usada como parâmetro e desta maneira aparecendo em todas as duplas de estímulos de cada alternativa, por exemplo, por dez vezes a nota musical DÓ foi o estímulo distrator, e por outras dez vezes a nota MI é que foi distratora, de forma ascendente (DÓ-RÉ OU RÉ-MI) ou descendente (RÉ-DÓ OU MI-RÉ) em relação à escala musical, sendo que em cada alternativa apresentava-se apenas um único par de notas por vez. Uma ilustração a respeito do procedimento geral de apresentação de estímulos pelo Psysounds é apresentada a seguir na Figura 1.

Os voluntários foram instruídos a falar a palavra "primeiro", indicando que o experimentador deveria pressionar 
Silva, J. A., Galdino, M. K. C., Simas, M. L. B. \& Santos, N. A. (2015). Consequências da Ingestão Moderada de Etanol na Discriminação de Notas Musicais.

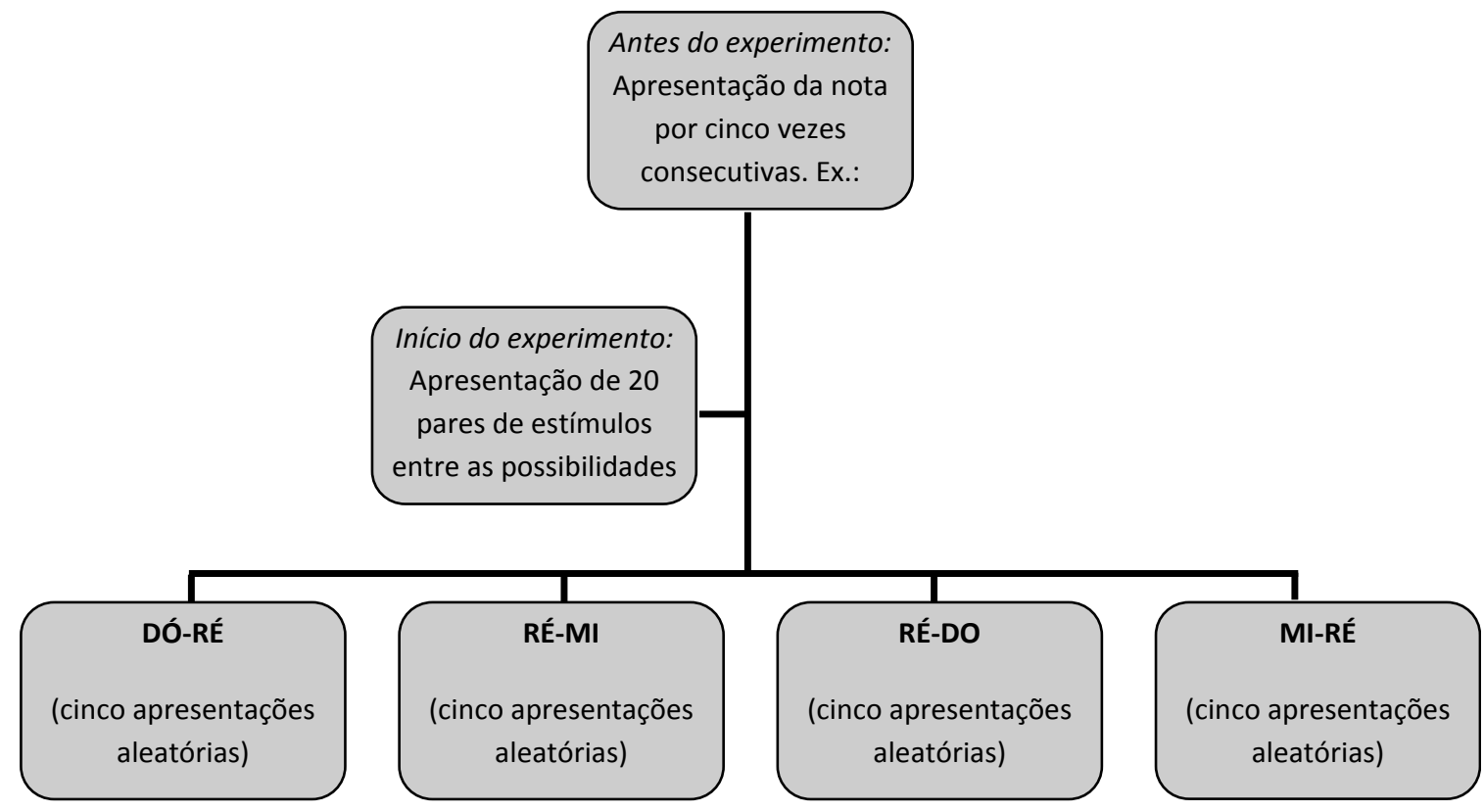

Figura 1. Procedimento de apresentação dos estímulos pelo Psysounds: exemplo em função da nota musical RÉ (ver explicação no texto).

o botão esquerdo do mouse, quando julgavam que a nota musical de teste tinha surgido em primeiro lugar no par de estímulos apresentados. Quando eles consideravam que a nota musical de teste era a segunda a ser apresentada no par de estímulos, deveriam dizer a palavra "segundo" e o experimentador, pressionar o botão direito do mouse. Antes que cada sessão tivesse início era verificado se o participante realmente havia entendido as instruções transmitidas. Confirmada a compreensão da tarefa pelos voluntários, começava-se o experimento. O próprio experimentador era quem apertava o botão do mouse para evitar que possíveis alterações motoras ocasionadas pela ingestão de álcool interferissem nos resultados dos participantes.

O mesmo grupo de voluntários passou pelas duas condições (ingestão de álcool e placebo) em dois dias diferentes em um espaço temporal de aproximadamente duas semanas. Foi utilizada a técnica do contrabalanceamento para controlar o efeito da ordem de ocorrência das condições para cada indivíduo. Todas as pessoas que foram convidadas a participar do estudo, e o aceitaram, foram solicitadas a não consumirem alimentos duas horas antes da pesquisa e a não ingerirem álcool nas 24 horas que a antecediam (ainda assim, a concentração de álcool no sangue foi aferida antes do início de cada sessão experimental). Na ocasião do teste, a quantidade de bebida alcoólica ingerida por cada participante (para a obtenção do valor de $0,08 \% \mathrm{BAC}$ ) foi conseguida por meio do software Dosagem.

A administração de álcool foi realizada sob a forma de vodka da marca "Stolichinaya", com concentração de $40 \%$, diluída em suco de maracujá, na proporção de 1:3. Já na condição placebo, foi feita a substituição da bebida alcoólica por duas colheres de sopa de suco de limão em pó. Uma quantidade de $10 \mathrm{ml}$ da vodka também foram acrescentadas para disfarçar o aroma da bebida.

Padronizou-se o tempo médio para ingestão do conteúdo em 15 minutos. O pico de álcool no sangue era alcançado em média com 30 minutos. O etilômetro foi utilizado para estimar o teor de álcool no sangue no início e ao fim da sessão experimental, bem como na chegada do participante ao laboratório, para confirmar se realmente o mesmo não havia ingerido álcool nas 24 horas antecedentes. A sessão experimental foi iniciada quando o teor de álcool sanguíneo alcançou a marca aproximada de $0,08 \%$ BAC.

\section{Resultados}

A alcoolemia na condição experimental atingiu o nível médio esperado de aproximadamente $0,08 \%$ BAC $(0,078 \pm 0,016)$. Ao final de cada experimento, o próprio programa PsySounds produziu uma folha de resposta apresentando os principais resultados obtidos. Entre os dados dispostos no relatório da sessão experimental dispunha-se a quantidade de acertos, que correspondeu ao número de vezes que o participante conseguiu detectar o estímulo sonoro de teste.

Estes índices de acertos conseguidos foram analisados nas condições (ou grupos) experimental (voluntários alcoolizados) e controle (voluntários não alcoolizados). A Tabela 2, a seguir, apresenta os valores brutos e as frequências médias de acertos no teste de discriminação das notas musicais para o conjunto de voluntários agrupados. Os valores diferenciam-se nas condições controle e experimental para cada uma das notas musicais avaliadas. Os índices apresentados devem ser considerados com base nas 800 respostas possíveis de cada nota musical testada (40 pessoas x 20 estímulos). 
Psicologia: Reflexão e Crítica, 28(1), 147-156.

Tabela 2

Número Total e Média das Respostas Corretas Obtidas no Teste de Discriminação Sonora para o Conjunto de Voluntários Agrupados nas Condições Experimental (álcool) e Controle (sem álcool) para as Notas Musicais RÉ, FÁ E LÁ

\begin{tabular}{lcccccc}
\hline & \multicolumn{2}{c}{ RÉ } & \multicolumn{2}{c}{ FÁ } & \multicolumn{2}{c}{ LÁ } \\
\cline { 2 - 6 } & $\begin{array}{c}\text { Respostas } \\
\text { Corretas }\end{array}$ & Média & $\begin{array}{c}\text { Respostas } \\
\text { Corretas }\end{array}$ & Média & $\begin{array}{c}\text { Respostas } \\
\text { Corretas }\end{array}$ & Média \\
\hline Álcool & 578 & $\begin{array}{c}14,45 \\
(D P=2,56)\end{array}$ & 565 & $\begin{array}{c}14,13 \\
(D P=3,53)\end{array}$ & 565 & $\begin{array}{c}14,13 \\
(D P=3,23)\end{array}$ \\
Sem Álcool & 603 & $\begin{array}{c}15,08 \\
(D P=2,98)\end{array}$ & 609 & $\begin{array}{c}15,22 \\
(D P=2,61)\end{array}$ & 580 & $\begin{array}{c}14,5 \\
(D P=2,75)\end{array}$ \\
\hline
\end{tabular}

Nota. $D P$ = Desvio-Padrão. A média de acertos foi obtida pelo somatório geral de respostas corretas para cada nota musical dividida pelo total de voluntários do estudo.

Como visto na Tabela 2, as médias de respostas corretas foram sempre maiores na condição em que os indivíduos não haviam ingerido álcool, sendo que esse número de acertos aumentou segundo as notas musicais na sequência LÁ, RÉ, E FÁ. Na condição em que os voluntários estavam alcoolizados, as médias de respostas certas das notas musicais foram iguais para as notas FÁ E LÁ.

Na nota musical RÉ, os voluntários tiveram 25 acertos a menos em comparação a eles mesmos quando estavam alcoolizados. Para a nota musical FÁ, foram 44 acertos a menos. Já para a nota LÁ, foram apenas 15 respostas a menos quando haviam ingerido etanol. Esses valores sugerem que o etanol altera a discriminação de notas musicais na sequência FÁ, RÉ E LÁ.

Aplicou-se o teste não-paramétrico $X^{2}$ (Qui-quadrado) de aderência para confirmação estatística dos resultados obtidos. Esse teste foi usado para a comparação entre as condições de ingestão e não ingestão alcoólica tomadas separadamente para cada uma das notas musicais utilizadas (RÉ, FÁ, LÁ). Considerou-se como referência o índice de acertos na discriminação das mesmas. A escolha desses testes se deu tanto pelos valores calculados serem números inteiros, sendo assim variáveis discretas, quanto por se tratarem de frequências de acertos obtidas por meio de um teste dicotômico de acertos e erros.

A comparação estatística entre as condições experimental e controle das respostas dos voluntários agrupados, por meio de um $X^{2}$ de aderência, apresentou diferença significante para todas as notas musicais utilizadas - RÉ $\left(X^{2}=201,069 ; g l=12 ; p=0,001\right), \mathrm{FÁ}\left(X^{2}=268,821 ; g l=\right.$ $13 ; p=0,001)$ e LÁ $\left(X^{2}=154,774 ; g l=12 ; p=0,001\right)-$ ao nível de significância de $p<0,05$. O V de Cramer, que trata sobre a correlação entre as variáveis "ingestão de etanol" e "notas musicais" apresentou os seguintes valores: RÉ $=$ 0,$41 ; \mathrm{FÁ}=0,48$; e LÁ $=0,36$, indicando a existência de uma relação, ainda que fraca, entre as variáveis em questão.

Esses resultados sugerem que os voluntários responderam de forma diferenciada para as duas condições estabelecidas no referente estudo. Esses dados podem ser visualizados na Figura 2, a seguir. As barras finas e pretas verticais representam o erro padrão da média de acertos para a discriminação entre as notas musicais.

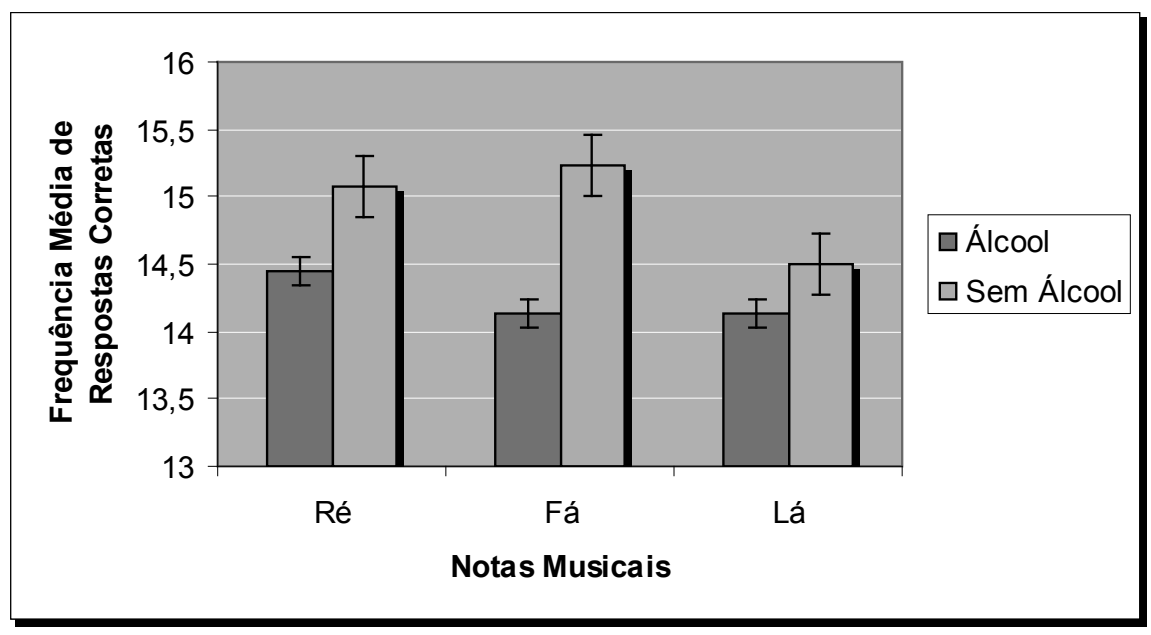

Figura 2. Discriminação das notas musicais pelos voluntários $(n=40)$ nas condições experimental (álcool) e controle (sem álcool). Os resultados foram significantes para as três frequências sonoras utilizadas 
Silva, J. A., Galdino, M. K. C., Simas, M. L. B. \& Santos, N. A. (2015). Consequências da Ingestão Moderada de Etanol na Discriminação de Notas Musicais.

Como observado na Figura 2, os valores para a discriminação das notas musicais tiverem um índice de acertos superior para a condição na qual os indivíduos não ingeriam álcool. Esses resultados sugerem uma diminuição na discriminação de todas as notas musicais utilizadas no estudo após a ingestão de etanol, sendo que a maior discriminação é percebida para a nota musical FÁ, seguido pelas notas RÉ E LÁ.

\section{Discussão}

O objetivo deste estudo foi verificar os efeitos da ingestão de etanol na discriminação das notas musicais RÉ (293,7 Hz), FÁ (349,2 Hz) e LÁ (440,0 Hz) na quarta oitava do piano, utilizando como parâmetro a concentração alcoólica sanguínea de $0,08 \%$. Para tanto, foi criado e utilizado um instrumento que testa a discriminação das notas musicais em adultos jovens após a ocorrência de procedimentos experimentais padrões para a ingestão do etanol.

Os resultados obtidos confirmaram as hipóteses iniciais de que as respostas dos indivíduos após a ingestão de etanol seriam afetadas negativamente. O presente estudo detectou que os voluntários se diferenciaram em suas respostas antes e após a ingestão de etanol em todas as notas musicais (ver Figura 2). Assim, os resultados sugerem que existem alterações na discriminação auditiva para notas musicais consequentes ao uso de bebidas alcoólicas.

Ainda que uma série de estudos relacionados aos vários atributos do sistema auditivo sob o efeito do álcool possa ser encontrada (He et al., 2013; Jääskeläinen et al., 2000; Kahkonen et al., 2005; Pearson, 1997; Pearson et al., 1999), considera-se ainda escassa a quantidade de pesquisas psicofísicas existentes no que diz respeito ao processamento psicológico das frequências sonoras significativas socialmente. A compreensão dos estímulos sonoros, principalmente dos tons complexos tipicamente utilizados em música tradicional, da captação externa ao processamento mais central no encéfalo tem sido um objeto de difícil controle, o que relativamente tem acarretado resultados não conclusivos nos estudos encontrados (Oliveira, Canedo, \& Rossato, 2002; Overath et al., 2007).

Segundo McDermott e Oxenham (2008), o estudo dos atributos musicais (por exemplo, de habilidades musicais cognitivas e motoras como tocar instrumentos e reconhecer composições ou ainda discriminar propriedades psicoacústicas como timbres ou contornos melódicos) é relevante para a construção do conhecimento relativo aos aspectos sócio-clínicos e neurobiológicos ligados à audição. Para Pearson (1997), a capacidade de discriminação de frequências sonoras é especialmente importante quando se lida com sinais auditivos complexos, tais como a capacidade de entender a palavra falada, bem como é crucial para a localização das fontes sonoras. Estas habilidades auditivas podem, então, atuar como facilitadoras em tarefas de interação social, a exemplo dos processos comunicativos verbais (Oxenham, Fligor, Mason, \& Kidd, 2003).
Um dos modelos de processamento da audição animal encontra-se no estudo de Sloan, Dodd, e Rennaker (2009), no qual compararam a discriminação de frequências por ratos albinos. Eles demonstraram melhora no desempenho médio dos sujeitos com o aumento da frequência de referência, sendo que a mudança ascendente da frequência foi acompanhada pelo aumento da quantidade de acertos nas tarefas de discriminação sonora. Deve-se considerar que o estudo de Sloan et al., além de utilizar animais como sujeitos, empregou tons puros e bem abaixo dos valores de frequência usados neste manuscrito (de 2,31 a $27,7 \mathrm{~Hz}$ ). Dessa forma, não é possível realizar uma comparação direta entre os dois estudos devido às diferenças inerentes aos animais e humanos, a exemplo das estruturas anatomofisiológicas e dos ambientes contextuais díspares entre eles. Contudo, os modelos animais são importantes para a criação de hipóteses iniciais a respeito do funcionamento neuro-sensorial dos indivíduos e dessa forma, tem sido utilizado para o entendimento inicial de vários comportamentos humanos.

Os animais podem ser treinados para classificar frequências como mais altas ou mais baixas que determinado tom de referência, essa tarefa pode ser mais difícil para os animais, admitindo que eles sejam submetidos cotidianamente e tenham que lidar com estímulos muito menos complexos que os humanos. Isso foi demonstrado no estudo comparativo entre a discriminação de frequências sonoras para furões e humanos realizado por Walker, Schnupp, Hart-Schnupp, King, e Bizley (2009). Deste modo, é de se esperar que os padrões de processamento de estímulos padrões sejam diferenciados para animais e humanos.

Os indivíduos são capazes de identificar características distintas do som dentro da faixa que corresponde ao seu espectro audível, podendo discriminar diferentes tons, reconhecendo, por exemplo, uma nota musical como diferente de outra (Dias, 2009). Segundo Pearson (1997), em seu estudo no qual testava o limiar auditivo, a ingestão de álcool a $0,08 \%$ BAC alterou negativamente apenas a habilidade de identificação das frequências mais altas (acima de $1.000 \mathrm{~Hz}$ ). Ainda que não se tenha trabalhado com limiares auditivos, mas sim com a discriminação entre frequências de tons complexos (notas musicais na quarta oitava), os resultados do presente manuscrito podem estar relacionados aos de Pearson. Na região relativamente médio-aguda e de alta sensibilidade da audição humana, examinada em ambos estudos, as alturas podem ser muito bem relacionadas perceptualmente umas com as outras.

Neste estudo encontrou-se diferença estatisticamente significante para todas as notas musicais utilizadas (RÉ, FÁ E LÁ) quando se comparou as condições controle (ingestão de placebo) e experimental (ingestão da bebida alcoólica). E ainda que as associações entre as variáveis "ingestão de etanol" e "notas musicais" não tenham se mostrado tão expressivas como pode ser visto pelos valores do $\mathrm{V}$ de Cramer, isso confirma de alguma forma a sugestão de Pearson (1997) de que as tarefas de discriminação sonora são mais prejudicadas pelos efeitos do álcool. Ratifica 
também estudos neurológicos como o de Jääskeläinen et al. (2000), que apresentou supressão auditiva transitória, demonstrada em exames de eletroencefalografia, ocasionada pela ingestão de álcool à $0,05 \% \mathrm{BAC}$ em uma tarefa de atenção seletiva.

O estudo de Pihkanen e Kauko (1962) analisou as consequências do álcool para a discriminação auditiva de frequências sonoras utilizando três pessoas com deficiência visual que eram afinadoras de pianos. Dois, dos três voluntários, apresentaram uma diminuição estatisticamente significante da sua capacidade de discriminar frequências, o que apóia os achados do presente trabalho. No entanto, não foram relatadas informações a respeito das frequências utilizadas. Além disso, devido à utilização de uma amostra pequena e especializada, foi impossível realizar o contrabalanceamento da ingestão alcoólica (alternar aleatoriamente a inoculação de bebida alcoólica entre os voluntários), como foi feito no presente estudo para evitar os efeitos de ordem.

Os resultados encontrados neste estudo foram avaliados em termos de mecanismos plausíveis pelos quais o álcool pode agir no organismo (Pearson, 1997). Esses dados obtidos não podem ser totalmente creditados unicamente as ações do álcool em um único mecanismo. Parece que uma explicação mais completa dos efeitos do álcool no processamento sensorial requer um modelo que inclui mais de um mecanismo fisiológico e consequentemente comportamental para os diversos indivíduos. Estimulações diretas do Núcleo Geniculado Medial e dos nervos auditivos, por exemplo, resultam em respostas do córtex auditivo claramente diminuídas após uso de etanol (Omar, Hailstone, Warren, Crutch, \& Warren, 2010; Teo \& Ferguson, 1986).

Investigações em relação aos efeitos do álcool sobre os mecanismos de inibição no sistema auditivo têm se mostrado importantes. Nesse sentido, sugere-se que o etanol atue de forma inibitória sobre os mecanismos fisiológicos das células aferentes, reduzindo a excitação promovida pela presença dos estímulos auditivos. Assim, como para se chegar a um nível mínimo de atividade neural em um determinado período de tempo, a amplitude relativa das respostas neurais depende da frequência do som, o processamento auditivo é prejudicado (Pearson, 1997). Por um lado, o presente estudo não pretende propor um modelo neurobiológico para as respostas comportamentais supostamente encontradas. Por outro, os mecanismos neurobiológicos podem auxiliar na compreensão das mesmas.

Resultados diferentes e por vezes contraditórios podem ser encontrados para experimentos de ingestão alcoólica, constatando inclusive que o álcool pode ter efeitos diferenciados em diversas frequências sonoras, alterando ou não a percepção das mesmas, o que podem ser explicado de alguma maneira pelas distintas formas com que são feitos o controle e a medida da concentração alcoólica (Carpenter, 1962; Eggleton, 1941; Himwich, 1957; Hogan \& Linfield, 1983; Levine, Kramer, \& Levine, 1975; Wallgren \& Barry, 1970).
Neste aspecto, foram utilizadas neste estudo adaptações das formulações padronizadas por Brick (2006) por meio do programa Dosagem, que auxiliou para que o teor alcoólico pretendido para as sessões experimentais fossem alcançadas em todas as ocasiões e por tempo suficiente para a execução dos testes necessários. À validade dos resultados encontrados agrega-se ainda ao fato do desenho do estudo ter sido intragrupal, ou seja, de que os voluntários tenham sido comparados com eles mesmos, o que ofereceu a oportunidade de obtenção de resultados mais precisos em relação às diferenças de condições experimentais planejadas para os procedimentos metodológicos utilizados neste estudo.

\section{Considerações Finais}

A caracterização do uso do álcool pela sociedade é relevante pelos seus aspectos epidemiológicos e biopsicossociais. Os prejuízos acarretados aos indivíduos pelo consumo do álcool estão relacionados diretamente as questões de saúde pública, o que reafirma a abrangência dos possíveis efeitos que a ingestão do etanol pode ocasionar. Deste estudo foi possível concluir que a ingestão do etanol presente nas bebidas alcoólicas pode alterar de alguma maneira a discriminação das notas musicais e consequentemente das frequências sonoras auditivas dentro da faixa testada.

De forma geral, diferenças de resultados entre os estudos existentes sobre audição e ingestão alcoólica podem estar conexas aos distintos aspectos metodológicos, aos critérios de seleção da amostra, aos tipos de instrumentos e de estímulos utilizados, entre outros motivos. Esse estudo pretendeu iniciar uma série de investigações em percepção auditiva, utilizando a discriminação de notas musicais para verificar o desempenho de adultos jovens em diversas condições. Entre as quais podem ser incluídos faixa etária, patologias físicas, deficiências, transtornos psicológicos, e contato com diferentes substâncias tóxicas.

Além do uso dessas variáveis, outras sugestões para estudos posteriores são o uso da opção de tempo de reação proporcionado pelo PsySounds, o aumento do número de voluntários na amostra, e o uso de outras notas musicais na mesma oitava utilizada ou em diferentes oitavas, como forma de caracterizar o processamento auditivo de variadas frequências de som. O uso de diferentes oitavas ou registros (isto é, subgrave, grave, agudo, superagudo, etc.) possibilitará a estimulação de distintas áreas da cóclea (ligadas às baixas, médias e altas frequências sonoras), onde possam ser encontradas alterações decorrentes de qualquer variável experimental pretendida. Por se tratar de um estudo exploratório as outras regiões de frequências da faixa audível humana não foram testadas nesse momento, porém, estas serão certamente testadas em próximas pesquisas. 
Silva, J. A., Galdino, M. K. C., Simas, M. L. B. \& Santos, N. A. (2015). Consequências da Ingestão Moderada de Etanol na Discriminação de Notas Musicais.

\section{Referências}

Bastos, F. I., Caiaffa, W., Rossi, D., Vila, M., \& Malta, M. (2007). The children of Mama Coca: Coca, cocaine and the fate of harm reduction in South America. International Journal of Drug Policy, 18, 99-106. doi:10.1016/j.drugpo.2006.11.017

Brick, J. (2006). Standardization of alcohol calculations in research. Alcoholism: Clinical and Experimental Research, 30, 1276-1287. doi:10.1111/j.1530-0277.2006.00155.x

Carpenter, J. A. (1962). Effects of alcohol on some psychological processes. Quarterly Journal of Studies on Alcohol, 23, 274-314. Retrieved from http://www.safetylit.org/citations/ index.php? fuseaction $=$ citations.viewdetails\& citationIds []$=\mathrm{c}$ itjournalarticle $82133 \quad 1$

Cavalcanti, M. K., \& Santos, N. A. (2008). Alterações na sensibilidade ao contraste relacionadas à ingestão de álcool. Psicologia: Teoria e pesquisa, 24, 515-518. Recuperado em http://www.scielo.br/pdf/ptp/v24n4/15.pdf

Cornsweet, T. N. (1970). Visual perception. New York: Academic Press. Retrieved from http://books.google.com.br/ books?hl=pt-BR\&lr=\&id=ubaGAAAAQBAJ\&oi=fnd\&pg= PP1\&ots=TByltwXK14\&sig=zouWcKuSkg1u-jVX3yh2p9 $\mathrm{dtV} 8 \&$ redir_esc $=\mathrm{y} \# \mathrm{v}=$ onepage $\& \mathrm{q} \& \mathrm{f}=$ false

Dias, C. A. S. (2009). Investigação das funções musicais em sujeito afásicos (Dissertação de mestrado em Distúrbios da Comunicação, Universidade Tuiuti do Paraná, Curitiba, PR, Brasil). Recuperado em http://meloteca.com/musicoterapia2014/investigacao-das-funcoes-musicais-em-sujeitos-afasicos.pdf

Eggleton, M. G. (1941). The effect of alcohol on the central nervous system. British Journal of Psychology, 32, 52-61. doi:10.1111/j.2044-8295.1941.tb01009.x

Engels, R. C. M. E., Poelen, E. A. P., Spijkerman, R., \& Ter Bogt, T. (2012). The effects of music genre on young people's alcohol consumption: An experimental observational study. Substance Use and Misuse, 47, 180-188. doi:10.3109/1082 6084.2012 .637461

Galdino, M. K. C., Mendes, L. C., Vieira, J. G., Simas, M. L. B., \& Santos, N. A. (2011). Percepção visual de grade senoidal radial após o consumo de álcool. Psicologia USP, 22, 99-115. doi:10.1590/S0103-65642011005000001

Galdino, M. K. C., Silva, J. A., Santos, N. A., \& Simas, M. L. B. (2010). O efeito da ingestão de álcool na percepção visual: Revisão sistemática. Neurobiologia, 73, 151-161. Recuperado em www.neurobiologia.org

He, J. B., Li, B. B., Guo, Y. Y., Naatanen, R., Pakarinen, S., \& Luo, Y. J. (2013). Effects of alcohol on auditory pre-attentive processing of four sound features: Evidence from mismatch negativity. Psychopharmacology, 225, 353-360. doi:10.1007/ s00213-012-2816-8.

Himwich, H. E. (1957). The physiology of alcohol. The Journal of the American Medical Association, 163, 545-549. doi:10.1001/jama.1957.82970420003009

Hogan, R. E., \& Linfield, P. B. (1983). The effects of moderate doses of ethanol on heterophoria and other aspects of binocular vision. Ophthalmic and Physiological Optics, 3, 21-31. doi:10.1111/j.1475-1313.1983.tb00576.x

Jääskeläinen, I. P., Hirvonen, J., Saher, M., Pekkonen, E., Sillanaukee, P., Näätänen, R., \& Tiitinen, H. (2000). Dose-dependent suppression by ethanol of transient auditory 40-Hz response. Psychopharmacology, 148, 132-135. Retrieved from https://tuhat.halvi.helsinki.fi/portal/en/publi- cations/dosedependent-suppr\%2868ba0518-97a1-4078-bde9$-35 \mathrm{c} 5 \mathrm{f} 0 \mathrm{bcdf} 2 \mathrm{~b} \% 29 . \mathrm{html}$

Kahkonen, S., Rossi, E. M., \& Yamashita, H. (2005). Alcohol impairs auditory processing of frequency changes and novel sounds: A combined MEG and EEG study. Psychopharmacology, 177, 366-372. doi:10.1007/s00213-004-1960-1

Kornreich, C., Brevers, D., Canivet, D., Ermer, E., Naranjo, C., Constant, E., ...Noel, X. (2013). Impaired processing of emotion in music, faces and voices supports a generalized emotional decoding deficit in alcoholism. Addiction, 108 , 80-88. doi:10.1111/j.1360-0443.2012.03995.x

Levine, J. M., Kramer, G. G., \& Levine, E. N. (1975). Effects of alcohol on human performance: An integration of research findings based on an abilities classification. Journal of Applied Psychology, 60, 285-293. Retrieved from http://psycnet.apa. org/journals/apl/60/3/285.pdf

Levitt, H. (1971). Transformed up-down methods in psychophysics. Journal of the Acoustical Society of America, 49, 467-477. Retrieved from http://bdml.stanford.edu/twiki/pub/Haptics/ DetectionThreshold/psychoacoustics.pdf

McDermott, J. H., \& Oxenham, A. J. (2008). Music perception, pitch, and the auditory system. Current Opinion in Neurobiology, 18, 452-463. doi:10.1016/j.conb.2008.09.005

Oliveira, J. A. A., Canedo, D. M., \& Rossato, M. (2002). Otoproteção das células ciliadas auditivas contra a ototoxidade da amicacina. Revista Brasileira de Otorrinolaringologia, 68 , 7-14. doi:10.1590/S0034-72992003000200012

Omar, R., Hailstone, J. C., Warren, J. E., Crutch, S. J., \& Warren, J. D. (2010). The cognitive organization of music knowledge: A clinical analysis. Brain, 133, 1200-1213. Retrieved from http://www.ncbi.nlm.nih.gov/pmc/articles/PMC2850578/

Overath, T., Cusack, R., Kumar, S., von Kriegstein, K., Warren, J. D., Grube, M., ...Griffiths, T. D. (2007). An information theoretic characterization of auditory encoding. Plos Biology, 5, 2723-2732. doi:10.1371/journal.pbio.0050288

Oxenham, A. J., Fligor, B. J., Mason, C. R., \& Kidd, G., Jr. (2003). Informational masking and musical training. Journal of the Acoustical Society of America, 114, 1543-1549. Retrieved from http://www.ncbi.nlm.nih.gov/pubmed/14514207

Pearson, P. M. (1997). The effects of ethyl alcohol on visual and auditory thresholds (Doctoral dissertation, University of Western Ontario, London). Retrieved from http://www.scielo. br/scielo.php?script $=$ sci nlinks\&ref $=000068 \&$ pid $=$ S0102 $-3772200800040001500007 \& \operatorname{lng}=\mathrm{en}$

Pearson, P., Dawe, L. A., \& Timney, B. (1999). Frequency selective effects of alcohol on auditory detection and frequency discrimination thresholds. Alcohol \& Alcoholism, 34, 741-749. doi:10.1093/alcalc/34.5.741

Piccinelli, M., Tessari, E., Bortolomasi, M., Piasere, O., Semenzin, M., Garzotto, N., \& Tansella, M. (1997). Efficacy of the alcohol use disorders identification test as a screening tool for hazardous alcohol intake and related disorders in primary care: A validity study. British Medical Journal, 314, 420-424. Retrieved from http://www.ncbi.nlm.nih.gov/pubmed/9040389

Pihkanen, T., \& Kauko, O. (1962). The effects of alcohol on the perception of musical stimuli. Annales Experimentalis et Biologiae Fenniae, 40, 275-285.

Silva, J. A, Galdino, M. K. C., Gadelha, M. J. N., Andrade, M. J. O., \& Santos, N. A. (2013). Revisão sobre o processamento neuropsicológico dos atributos tonais da música no contexto ocidental. Avances en Psicología Latinoamericana, 31, 86-96. Recuperado em http://www.scielo.org.co/scielo.php?pid=S1 $79447242013000100007 \&$ script $=$ sci arttext 
Sloan, A. M., Dodd, O. T., \& Rennaker, R. L. (2009). Frequency discrimination in rats measured with tone-step stimuli and discrete pure tones. Hearing Research, 251, 60-69. Retrieved from http://www.ncbi.nlm.nih.gov/pmc/articles/ PMC2670949/

Teo, R. K., \& Ferguson, D. A. (1986). The acute effects of ethanol on auditory event-related potentials. Psychopharmacology (Berlin), 90, 179-184. Retrieved from http://www.ncbi.nlm. nih.gov/pubmed/2878457

Vieira, R. M. T., Serafim, A. P., \& Saffi, F. (2007). Prejuízos neurocognitivos na dependência alcoólica: Um estudo de caso. Revista de Psiquiatria Clínica, 34, 246-250. Recuperado em http://www.hcnet.usp.br/ipq/revista/vol34/n5/246.html

Wallgren, H., \& Barry, H., III. (1970). Actions of alcohol: Vol. 1. Biochemical, physiological and psychological aspects. New York: Elsevier.

Walker, K. M. M., Schnupp, J. W. H., Hart-Schnupp, S. M. B., King, A. J., \& Bizley, J. K. (2009). Pitch discrimination by ferrets for simple and complex sounds. Journal of the Acoustical Society of America, 126, 1321-1335. Retrieved from http://ora.ox.ac.uk/objects/uuid:313f8f6e-0461-4965-b61b-bfde6b5911db 